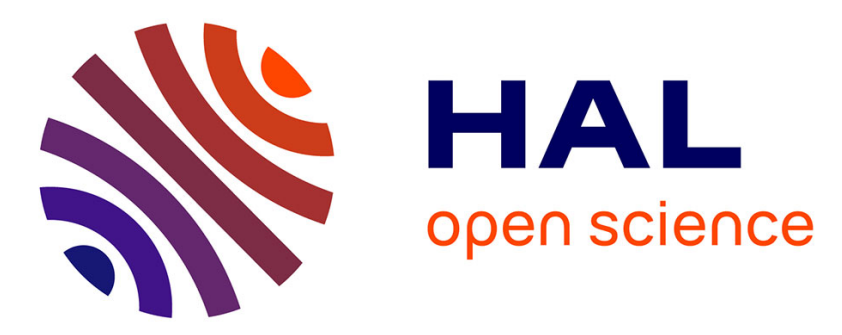

\title{
Boundary Conditions for an Ocean Related System with a Small Parameter
}

\author{
Antoine Rousseau, Roger M. Temam, Joe Tribbia
}

\section{To cite this version:}

Antoine Rousseau, Roger M. Temam, Joe Tribbia. Boundary Conditions for an Ocean Related System with a Small Parameter. American Mathematical Society, 2005, Contemporary Mathematics, Nonlinear Partial Differential Equations and Related Analysis, 371. inria-00172515

\section{HAL Id: inria-00172515 https://hal.inria.fr/inria-00172515}

Submitted on 17 Sep 2007

HAL is a multi-disciplinary open access archive for the deposit and dissemination of scientific research documents, whether they are published or not. The documents may come from teaching and research institutions in France or abroad, or from public or private research centers.
L'archive ouverte pluridisciplinaire $\mathbf{H A L}$, est destinée au dépôt et à la diffusion de documents scientifiques de niveau recherche, publiés ou non, émanant des établissements d'enseignement et de recherche français ou étrangers, des laboratoires publics ou privés. 
To appear in

"Nonlinear PDEs and Related Analysis"

Contemporary Mathematics, AMS.

\title{
BOUNDARY CONDITIONS FOR AN OCEAN RELATED SYSTEM WITH A SMALL PARAMETER
}

\author{
A. ROUSSEAU ${ }^{b}$, R. TEMAM ${ }^{b *}$, AND J. TRIBBIA ${ }^{\sharp}$
}

\begin{abstract}
A linear system derived from the Primitive Equations (PEs) of the atmosphere and the ocean is considered. Existence and uniqueness of solutions, behavior as a small viscosity parameter tends to zero are studied for different boundary conditions ; one of the boundary condition is of Dirichlet type and it produces reflections of waves at the boundary, the other one is of transparent type. Computational issues are addressed in a companion paper [RTT04], which also contains more details on the derivation of the system that we study here.
\end{abstract}

\section{INTRODUCTION}

The Primitive Equations of the ocean and the atmosphere are fundamental equations of geophysical fluid mechanics ([Ped87],[WP86],[Sal98]). In the presence of viscosity, it has been shown, in various contexts, that

2000 Mathematics Subject Classification: 35L50, 76N20, 76A02, 86A05.

NSF GRANTS: DMS-0074334 and DMS-0305110 
these equations are well-posed (see e.g. [LTW92a],[LTW92b], and the review article [TZ03]).

In the absence of viscosity, it is known that the PEs are not wellposed for any set of boundary conditions of local type (see [OS78] and also [TT03]). This difficulty is analyzed in [TT03] using a modal analysis in the vertical direction. To overcome this difficulty a modification of the PEs was proposed in [TT03], for which a set of local boundary conditions also given in this reference produces a decay of energy (in the absence of forcing).

The modified PEs introduced in [TT03] contain an added friction term $\delta w$ in the hydrostatic equation, so that the model is actually nonhydrostatic; here we call this system the $\delta$-PEs equations. Partial results of well-posedness of the $\delta$-PEs were proven and full wellposedness is conjectured at least in dimension two; these questions will be addressed elsewhere.

In a companion article [RTT04], we showed how to derive the system (0.1) below from the $\delta$-PEs after linearization of the $\delta$-PEs equations around a stratified flow with a constant velocity $\bar{U}_{0}$ in the (west-east) direction $O x$ and after performing a modal decomposition of the dependant unknowns in the vertical direction. Dropping the indices $n$, we obtain for the $n$-th mode $u_{n}, \psi_{n}$,

$$
\left\{\begin{array}{l}
u_{t}+\bar{U}_{0} u_{x}+\psi_{x}-\frac{\delta g}{N^{2} \lambda^{2}} u_{x x}=0 \\
\psi_{t}+\bar{U}_{0} \psi_{x}+\frac{1}{\lambda^{2}} u_{x}
\end{array}\right.
$$

where $(u, v)$ is the horizontal velocity, and $\psi$ is proportional to the temperature. The equation for $v$ is omitted here because it does not raise any additional difficulty; the coupling between $u$ and $v$ has been suppressed by setting the Coriolis parameter equal to 0 .

Here $\lambda=\lambda_{n}>0$ is the $n$-th eigenvalue in the vertical decomposition. We only consider the subcritical case where $\bar{U}_{0}<1 / \lambda$, the supercritical case being easier to solve; $g>0$ is the gravity constant and $N>0$ is a parameter related to the background stratified state. Solutions independent of the $y$ (south-north) variable are considered, and the Coriolis term which is not significant here is neglected so that the equation for the second component $v$ of the velocity is decoupled from the system (0.1). The system (0.1) is supplemented with the physically natural boundary conditions described below in (1.2) which are of Dirichlet type. 
The primary motivation of the article [RTT04] and the present one is to study the effect on such a mode of the added viscosity $\delta>0$. Although the limit of system $(0.1)$ is very simple as $\delta \longrightarrow 0$ (in particular after diagonalization), the numerical simulations reported in [RTT04] surprisingly showed that the boundary conditions of the limit system are not those expected. Our aim in Section 1 is to study the wellposedness of the perturbed problem and of the limit problem as $\delta$ (or $\varepsilon=\delta g / 2 N^{2} \lambda^{2}$ ) goes to zero; and the convergence of the solution with an explicit derivation of the correctors for the corresponding boundary layer.

A drawback of the system (0.1) in the physical context it was introduced, is that it produces a non physical reflection of waves at the boundary (see below and the discussion in [RTT04]). Hence in Section 2 we propose another set of boundary conditions for (0.1), inspired by the concept of transparent boundary conditions (TBC) and which does not produce reflection at the boundary, see (2.9)-(2.10). We prove that the problem is also well-posed, we provide a proof of convergence as $\delta$ (or $\varepsilon=\delta g / 2 N^{2} \lambda^{2}$ ) goes to zero to a natural limit system made up with uncoupled scalar transport equations (see (2.3)).

Finally in Section 3 we propose another set of transparent boundary conditions for (0.1), less satisfactory from the theorical viewpoint, but numerically more efficient, in fact the system used in the numerical simulations of [RTT04] (see the details in Section 3).

From the mathematical viewpoint the system that we consider here is a linear incompletely parabolic perturbation of a hyperbolic system. The problem of finding an appropriate set of boundary conditions for such systems has been addressed by a number of authors, with the purpose of obtaining a well-posed initial boundary value problem, or with the purpose of finding nonreflecting boundary conditions or both. Early papers devoted in general to the (nonlinear) compressible NavierStokes equations or the Shallow Water equations are those of Lions and Raviart [LR66], Strikwerda [Str77], Gustafsson and Kreiss [GK79], Rudy and Strikwerda [RS80, RS81], Michelson [Mic85]. More recent articles are e.g. those of Halpern and Schatzman [HS89], Halpern [Hal91] and Tourette [Tou97]. The problem of finding nonreflective boundary conditions in a general context is addressed in the classical articles of Engquist and Majda [EM77, EM79]. Section 1 of our article differs from the references above in that the boundary conditions for the 
incompletely parabolic system are known and derived from physical considerations and from the study in [TT03]. On the contrary in Sections 2 and 3, motivated by computational preoccupations, we look for nonreflective boundary conditions. To the best of our knowledge the issue of reflective and nonreflective boundary conditions for the Primitive Equations of the ocean and the atmosphere have not been addressed in the past.

\section{The systems With the Dirichlet BOUndary CONDitions}

In this section we consider the system (0.1) and the limit system obtained by setting formally $\varepsilon=0$. Both systems are supplemented with suitable boundary conditions. We show the well-posedness for each system and we then prove in Section 1.3 that when $\varepsilon$ goes to zero, the solution of (1.1) converges to the solution of (1.27) in relevant spaces, and with the boundary conditions presented below.

We start by presenting the two systems and studying their wellposedness.

\subsection{The $\varepsilon$-system.}

We are interested in studying the following system:

$$
\left\{\begin{array}{l}
u_{t}^{\varepsilon}+\bar{U}_{0} u_{x}^{\varepsilon}+\psi_{x}^{\varepsilon}-2 \varepsilon u_{x x}^{\varepsilon}=f, \\
\psi_{t}^{\varepsilon}+\bar{U}_{0} \psi_{x}^{\varepsilon}+\frac{1}{\lambda^{2}} u_{x}^{\varepsilon}=g .
\end{array} \quad 0<x<L, \quad t>0\right.
$$

with the following boundary conditions and initial data:

$$
\begin{gathered}
\left\{\begin{array}{l}
u^{\varepsilon}(0, t)=0, \\
\psi^{\varepsilon}(0, t)=0, \\
u^{\varepsilon}(L, t)=0,
\end{array}\right. \\
\left\{\begin{array}{l}
u^{\varepsilon}(x, 0)=u_{0}(x), \\
\psi^{\varepsilon}(x, 0)=\psi_{0}(x),
\end{array}\right.
\end{gathered}
$$

Remark 1.1. : For $\varepsilon>0$, the boundary conditions (1.2) are derived from the boundary conditions for a limited domain as proposed in [TT03]; namely: prescription of the normal velocity everywhere on the boundary and of the horizontal velocity and temperature on the parts of the boundary where the flow is incoming. Taking into account that the flow under consideration is a perturbation of the flow $\bar{U}_{0} e_{x}$, 
we find the boundary conditions (1.2): indeed $u^{\varepsilon}(0, t)$ and $u^{\varepsilon}(L, t)$ are the normal components of the velocity at $x=0$ and $L$, and $\psi^{\varepsilon}(0, t)$ corresponds to prescribing the temperature at $x=0$ where the flow is incoming.

For more convenience we will now write $(u, \psi)$ instead of $\left(u^{\varepsilon}, \psi^{\varepsilon}\right)$, so that (1.1) is the same as (0.1) with $\varepsilon=\delta g / 2 N^{2} \lambda^{2}$, the right-hand sides $f, g$ have been added for mathematical generality.

We now want to set the functional framework for (1.1)-(1.3). In the context of the linear semi-group theory ([Yos80], [Bré73], [Hen81], [Lio65], [Paz83]) the natural spaces and operators are the following:

$$
\begin{aligned}
& H=L^{2}(0, L) \times L^{2}(0, L) \\
& D(A)=\left\{U=(u, \psi) \in H, u_{x}, \psi_{x}, \text { and } u_{x x} \in L^{2}(0, L),\right. \\
& u(0)=\psi(0)=u(L)=0\} \\
& A\left(\begin{array}{l}
u \\
\psi
\end{array}\right)=\left(\begin{array}{l}
\bar{U}_{0} u_{x}+\psi_{x}-2 \varepsilon u_{x x} \\
\bar{U}_{0} \psi_{x}+\frac{1}{\lambda^{2}} u_{x}
\end{array}\right), \forall\left(\begin{array}{l}
u \\
\psi
\end{array}\right) \in D(A) .
\end{aligned}
$$

Then (1.1)-(1.3) can be written as follows:

$$
\frac{d U}{d t}+A U=F, \quad U(0)=U_{0}
$$

where $U_{0}=\left(u_{0}, \psi_{0}\right)^{T} \in D(A), U(t)=(u(t), \psi(t))^{T} \in D(A)$ for all time and $F=(f, g)^{T} \in H$.

To prove the well-posedness of this problem, we will use the HilleYosida theorem (see the references above):

Theorem 1.1. Let $H$ be a Hilbert space and let $A: D(A) \longrightarrow H$ be a linear unbounded operator, with domain $D(A) \subset H$. Assume the following:

(i) $D(A)$ is dense in $H$ and $A$ is closed,

(ii) $A$ is $\geq 0$, i.e. $(A U, U)_{H} \geq 0, \quad \forall U \in D(A)$,

(iii) $\forall \mu>0, A+\mu I$ is onto.

Then $-A$ is infinitesimal generator of a strongly continuous semigroup of contractions $\{S(t)\}_{t \geq 0}$, and for every $U_{0} \in H$ and $F \in L^{1}(0, T ; H)$, there exists a unique solution $U \in \mathcal{C}([0, T] ; H)$ of

$$
U(t)=S(t) U_{0}+\int_{0}^{t} S(t-s) F(s) d s,
$$


that is a weak form of (1.4).

If furthermore $(d U / d t)(0) \in H$ then ${ }^{1} U$ satisfies (1.4) and

$$
U \in \mathcal{C}([0, T] ; H) \cap L^{\infty}(0, T ; D(A)), \quad \frac{d U}{d t} \in L^{\infty}(0, T ; H) .
$$

The spaces $H, D(A)$, and the operator $A$ have been defined above. It is clear that $H$, supplemented with the inner product $(U, V)_{H}=$ $\int_{0}^{L}\left(U_{1} V_{1}+\lambda^{2} U_{2} V_{2}\right) d x$, is a Hilbert space. We will achieve the proof of well-posedness for the problem (1.4) within three steps, corresponding to the three main hypotheses of Theorem 1.1.

We start with (i):

Lemma 1.1. $D(A)$ is dense in $H$ and $A$ is a closed operator.

Proof. We write $D(A)=\left\{(u, \psi) \in\left(H^{2} \cap H_{0}^{1}\right) \times H_{l}^{1}\right\}$, where $H_{l}^{1}=\{\psi \in$ $\left.H^{1}(0, L), \psi(0)=0\right\}$.

To prove that $D(A)$ is dense in $H$, it is sufficient to observe that $\mathcal{C}_{0}^{\infty}(0, L)$ is dense in $L^{2}(0, L)$ since $\mathcal{C}_{0}^{\infty}(0, L) \times \mathcal{C}_{0}^{\infty}(0, L) \subset D(A)$.

To show that $A$ is closed, we consider a sequence $\left(u_{n}, \psi_{n}\right)=U_{n}$ of $D(A)$, such that:

$$
\begin{aligned}
U_{n} & \longrightarrow U \text { in } H, \\
A U_{n}=F_{n} & \longrightarrow F \text { in } H,
\end{aligned}
$$

and we want to verify that $U=(u, \psi) \in D(A)$ and $F=A U$, so that the graph of $A$ is closed.

By the definition of $A$

$$
\left\{\begin{aligned}
\bar{U}_{0} \frac{\partial u_{n}}{\partial x}+\frac{\partial \psi_{n}}{\partial x}-2 \varepsilon \frac{\partial^{2} u_{n}}{\partial x^{2}} & =f_{n} \\
\bar{U}_{0} \frac{\partial \psi_{n}}{\partial x}+\frac{1}{\lambda^{2}} \frac{\partial u_{n}}{\partial x} & =g_{n}
\end{aligned}\right.
$$

Hence, passing to the limit in the distribution sense we find

$$
\left\{\begin{aligned}
\bar{U}_{0} \frac{\partial u}{\partial x}+\frac{\partial \psi}{\partial x}-2 \varepsilon \frac{\partial^{2} u}{\partial x^{2}} & =f \\
\bar{U}_{0} \frac{\partial \psi}{\partial x}+\frac{1}{\lambda^{2}} \frac{\partial u}{\partial x} & =g .
\end{aligned}\right.
$$

\footnotetext{
${ }^{1}$ This is guaranteed if e.g. $U_{0} \in D(A)$ and $F$ is continuous (in $H$ ) at $t=0$.
} 
By combination of (1.9a) and (1.9b), we find:

$$
\left(\bar{U}_{0}^{2}-\frac{1}{\lambda^{2}}\right) \frac{\partial u_{n}}{\partial x}-2 \varepsilon \bar{U}_{0} \frac{\partial^{2} u_{n}}{\partial x^{2}}=\bar{U}_{0} f_{n}-g_{n} .
$$

We integrate on $(0, x)$, and obtain:

$$
\frac{\partial u_{n}}{\partial x}(x)=\frac{\partial u_{n}}{\partial x}(0)+\Phi\left(g_{n}-\bar{U}_{0} f_{n}\right)(x)
$$

where:

$$
\Phi(\varphi)(x)=\frac{1}{2 \varepsilon \bar{U}_{0}} \int_{0}^{x} \varphi\left(x^{\prime}\right) e^{-\left(1 / 2 \varepsilon \bar{U}_{0} \lambda^{2}-\bar{U}_{0} / 2 \varepsilon\right)\left(x-x^{\prime}\right)} d x^{\prime} .
$$

From the latter expression, we find that $\Phi: L^{2} \longrightarrow H^{1}$ is a linear continuous mapping.

Thanks to (1.8), it is clear that $\Phi\left(g_{n}-\bar{U}_{0} f_{n}\right)$ is bounded in $H^{1}(0, L)$. Differentiating (1.12), we then conclude that $\partial^{2} u_{n} / \partial x^{2}$ is bounded in $L^{2}$. Hence $u_{n}$ is bounded in $H^{2} \cap H_{0}^{1}$, and by (1.7), $u$ is in $H^{2}(0, L) \cap$ $H_{0}^{1}(0, L)$.

Similarly $(1.9 \mathrm{~b})$ shows that the sequence $\psi_{n}$ is bounded in $H_{l}^{1}$ and then by (1.7) $\psi$ is also in $H_{l}^{1}$. In conclusion $U=(u, \psi) \in D(A)$ and equations (1.10) show that $A U=F$, thus concluding the proof of Lemma 1.1.

To continue with the proof of well-posedness, we want to show that the operator $A$ is positive, that is:

Lemma 1.2. For every $U \in D(A),(A U, U)_{H} \geq 0$.

Proof. Let us compute the inner product, with any $U \in D(A)$ :

$$
\begin{aligned}
(A U, U)_{H}= & \int_{0}^{L}\left\{\left(\bar{U}_{0} u_{x}+\psi_{x}-2 \varepsilon u_{x} x\right) u+\left(\bar{U}_{0} \lambda^{2} \psi_{x}+u_{x}\right) \psi\right\} d x \\
= & \frac{1}{2} \bar{U}_{0}\left(u^{2}(L)-u^{2}(0)+\lambda^{2} \psi^{2}(L)-\lambda^{2} \psi^{2}(0)\right) \\
& -2 \varepsilon\left[u_{x} u\right]_{x=0}^{x=L}+2 \varepsilon \int_{0}^{L} u_{x}^{2} d x .
\end{aligned}
$$

Thanks to the boundary conditions (1.2), we find:

$$
(A U, U)_{H}=\frac{1}{2} \bar{U}_{0} \lambda^{2} \psi^{2}(L)+2 \varepsilon \int_{0}^{L} u_{x}^{2} d x \geq 0 .
$$

This shows that $A$ is a positive operator, and ends the proof of Lemma 1.2 
Remark 1.2. : One can derive from Lemma 1.2 a result of decrease of the energy of the system. If one defines :

$$
E(t)=\int_{0}^{L}\left(u^{2}(x, t)+\lambda^{2} \psi^{2}(x, t)\right) d x,
$$

then it is easy to show, following the proof of Lemma 1.2, that if $F=0$, any solution of $(1.4)$ in $L^{1}(0, T ; D(A))$ satisfies:

$$
\frac{d}{d t} E(t) \leq 0, \quad 0 \leq t \leq T
$$

We achieve the proof of well-posedness for (1.4) with the following lemma:

Lemma 1.3. The operator $A+\mu I$ is onto, $\forall \mu>0$.

Proof. Let $\mu$ be a positive real number, let $(f, g) \in H$; we look for $(u, \psi) \in D(A)$ such that:

$$
\left\{\begin{aligned}
\bar{U}_{0} u_{x}+\psi_{x}-2 \varepsilon u_{x x}+\mu u & =f \\
\bar{U}_{0} \psi_{x}+\frac{1}{\lambda^{2}} u_{x} & +\mu \psi=g .
\end{aligned}\right.
$$

with:

$$
\left\{\begin{array}{l}
u(0)=u(L)=0 \\
\psi(0)=0
\end{array}\right.
$$

From (1.17b), we find

$$
\psi_{x}+\frac{\mu}{\bar{U}_{0}} \psi=\frac{g}{\bar{U}_{0}}-\frac{1}{\bar{U}_{0} \lambda^{2}} u_{x}
$$

and integrating this equation we obtain:

$$
\psi=\Psi(g)-\frac{1}{\lambda^{2}} \Psi\left(u_{x}\right),
$$

where

$$
\Psi(\varphi)=\frac{1}{\bar{U}_{0}} \int_{0}^{x} \varphi\left(x^{\prime}\right) e^{-\mu\left(x-x^{\prime}\right) / \bar{U}_{0}} d x^{\prime} .
$$

The mapping $\Psi$ is similar to the mapping $\Phi$ defined in (1.13), and only some constants differ. In particular $u \in H^{1} \mapsto \Psi\left(u_{x}\right) \in H^{1}$ is continuous, and $\psi(g)$ is in $H^{1}$.

Thanks to (1.19) and (1.20), equations (1.17) and (1.18) are equivalent to finding $u \in H_{0}^{1}(0, L)$ such that

$$
-2 \varepsilon u_{x x}+\bar{U}_{0} u_{x}-\frac{1}{\lambda^{2}} \frac{d}{d x} \Psi\left(u_{x}\right)+\mu u=f-\frac{d}{d x} \Psi(g) .
$$


The variational formulation of (1.21) reads:

To find $u \in H_{0}^{1}(0, L)$, such that

$$
\begin{array}{r}
2 \varepsilon\left(u_{x}, \tilde{u}_{x}\right)+\bar{U}_{0}\left(u_{x}, \tilde{u}\right)+\frac{1}{\lambda^{2}}\left(\Psi\left(u_{x}\right), \tilde{u}_{x}\right)+\mu(u, \tilde{u}) \\
=\left(f-\frac{d}{d x} \Psi(g), \tilde{u}\right), \quad \forall \tilde{u} \in H_{0}^{1}(0, L) .
\end{array}
$$

We solve this problem using the Lax-Milgram theorem; the main point is to prove the coercivity on $H_{0}^{1}(0, L)$ of the bilinear form

$$
a(u, \tilde{u})=2 \varepsilon\left(u_{x}, \tilde{u}_{x}\right)+\bar{U}_{0}\left(u_{x}, \tilde{u}\right)+\frac{1}{\lambda^{2}}\left(\Psi\left(u_{x}\right), \tilde{u}_{x}\right)+\mu(u, \tilde{u}),
$$

and the continuity of

$$
l(\tilde{u})=\left(f-\frac{d}{d x} \Psi(g), \tilde{u}\right) .
$$

The latter is easy, observing that $f-d \Psi(g) / d x \in L^{2}$. Regarding the coercivity of $a$, it is sufficient to prove it for $u \in \mathcal{C}_{0}^{\infty}(0, L)$ since this space is dense in $H_{0}^{1}(0, L)$. For such a $u$ :

$$
a(u, u)=2 \varepsilon\left|u_{x}\right|_{L^{2}}^{2}+\frac{1}{\lambda^{2}}\left(\Psi\left(u_{x}\right), u_{x}\right)+\mu|u|_{L^{2}}^{2}
$$

From (1.20), we know that $\Psi\left(u_{x}\right)=\Psi_{0}$ is solution of:

$$
\left\{\begin{array}{l}
\bar{U}_{0} \frac{\partial \Psi_{0}}{\partial x}+\mu \Psi_{0}=u_{x} \\
\Psi_{0}(0)=0
\end{array}\right.
$$

Hence:

$$
\begin{aligned}
\left(\Psi_{0}, u_{x}\right) & =\left(\Psi_{0}, \bar{U}_{0} \frac{\partial \Psi_{0}}{\partial x}+\mu \Psi_{0}\right) \\
& =\mu\left|\Psi_{0}\right|_{L^{2}}^{2}+\frac{1}{2} \bar{U}_{0}\left(\Psi_{0}^{2}(L)-\Psi_{0}^{2}(0)\right) \\
& =\mu\left|\Psi_{0}\right|_{L^{2}}^{2}+\frac{1}{2} \bar{U}_{0} \Psi_{0}^{2}(L)
\end{aligned}
$$

Finally, inserting this in (1.24), we find:

$$
a(u, u) \geq 2 \varepsilon\left|u_{x}\right|_{L^{2}}^{2}+\mu|u|_{L^{2}}^{2}
$$

and the coercivity of $a$ is proven. Hence, thanks to Lax-Milgram theorem, there exists a unique solution $u \in H_{0}^{1}$ of (1.21). Thanks to (1.19), $\psi \in H^{1}$, and $\psi(0)=0$. Lastly, we obtain from (1.17a) that $u \in H^{2} \cap H_{0}^{1}$. The lemma is proven. 
All hypotheses have been proved and we thus infer from Theorem 1.1 the following result:

Theorem 1.2. Problem (1.1)-(1.3) is well-posed, that is for every $(f, g) \in L^{1}(0, T ; H)$, and every $\left(u_{0}, \psi_{0}\right) \in D(A)^{2}$, there exists a unique solution $U=(u, \psi)$ of (1.1)-(1.3) satisfying (1.6).

Remark 1.3. : We have proven that $-A$ is generator of a strongly continuous semigroup, which is sufficient for Theorem 1.2 (and Theorem 1.3 below). However $-A$ is not generator of an analytic semi-group and this would produce additional difficulties in the nonlinear case.

\subsection{The limit system $(\varepsilon=0)$.}

If we formally set $\varepsilon=0$ in (1.1), we obtain the following limit system:

$$
\left\{\begin{array}{l}
u_{t}^{0}+\bar{U}_{0} u_{x}^{0}+\psi_{x}^{0}=f, \\
\psi_{t}^{0}+\bar{U}_{0} \psi_{x}^{0}+\frac{1}{\lambda^{2}} u_{x}^{0}=g, \quad 0<x<L, \quad t>0 .
\end{array}\right.
$$

What was conjectured in [RTT04], based on the results of the numerical simulations, is that the boundary conditions for the limit system should be

$$
\left\{\begin{array}{l}
u^{0}(0, t)+\bar{U}_{0} \lambda^{2} \psi^{0}(0, t)=0, \\
u^{0}(L, t)=0 .
\end{array}\right.
$$

Of course, we impose the same initial data to $\left(u^{0}, \psi^{0}\right)$ :

$$
\left\{\begin{array}{l}
u^{0}(x, 0)=u_{0}(x), \\
\psi^{0}(x, 0)=\psi_{0}(x)
\end{array} \quad 0<x<L\right.
$$

We consider the change of variables which diagonalize (1.27), and we set

$$
\xi^{0}=u^{0}+\lambda \psi^{0}, \eta^{0}=u^{0}-\lambda \psi^{0} .
$$

In terms of $\xi^{0}, \eta^{0}$, equations (1.27)-(1.29) become

$$
\begin{aligned}
& \left\{\begin{array}{l}
\xi_{t}^{0}+\alpha \xi_{x}^{0}=f+\lambda g, \\
\eta_{t}^{0}-\beta \eta_{x}^{0}=f-\lambda g,
\end{array} \quad 0<x<L, t>0,\right. \\
& \left\{\begin{array}{l}
\left(\xi^{0}+\frac{\beta}{\alpha} \eta^{0}\right)(0, t)=0, \\
\left(\xi^{0}+\eta^{0}\right)(L, t)=0,
\end{array}\right.
\end{aligned}
$$

\footnotetext{
${ }^{2}$ And e.g. $(f, g)$ is continuous in $H$ at $t=0$; see footnote 1
} 


$$
\left\{\begin{array}{l}
\xi^{0}(x, 0)=\xi_{0}(x)=u_{0}(x)+\lambda \psi_{0}(x), \\
\eta^{0}(x, 0)=\eta_{0}(x)=u_{0}(x)-\lambda \psi_{0}(x), \quad 0<x<L,
\end{array}\right.
$$

where $\alpha=\bar{U}_{0}+1 / \lambda$, and $\beta=-\bar{U}_{0}+1 / \lambda$. We recall that we consider the subcritical case where $\bar{U}_{0}<1 / \lambda$, so that $\alpha>\beta>0$.

In the rest of this section we show the well-posedness of problem (1.27)-(1.29) (or equivalently (1.30)-(1.33)). Then, in Section 1.3, we do prove that $\left(u^{\varepsilon}, \psi^{\varepsilon}\right)$ converges to $\left(u^{0}, \psi^{0}\right)$ as $\varepsilon \longrightarrow 0$.

For (1.27)-(1.29), the natural functional framework is as follows:

$$
\begin{aligned}
& H=L^{2}(0, L) \times L^{2}(0, L), \\
& D\left(A^{0}\right)=\left\{U^{0}=\left(u^{0}, \psi^{0}\right) \in H, u_{x}^{0}, \psi_{x}^{0} \in L^{2}(0, L),\right. \\
& \left.u^{0}(0)+\bar{U}_{0} \lambda^{2} \psi^{0}(0)=u^{0}(L)=0\right\}, \\
& A^{0}\left(\begin{array}{c}
u^{0} \\
\psi^{0}
\end{array}\right)=\left(\begin{array}{c}
\bar{U}_{0} u_{x}^{0}+\psi_{x}^{0} \\
\bar{U}_{0} \psi_{x}^{0}+\frac{1}{\lambda^{2}} u_{x}^{0}
\end{array}\right), \quad \forall\left(\begin{array}{c}
u^{0} \\
\psi^{0}
\end{array}\right) \in D(A) .
\end{aligned}
$$

Equation (1.27) can then be written as follows:

$$
\frac{d U^{0}}{d t}+A^{0} U^{0}=F, \quad U^{0}(0)=U_{0},
$$

where $U_{0}=\left(u_{0}, \psi_{0}\right)^{T} \in D\left(A^{0}\right), U^{0}(t)=\left(u^{0}(t), \psi^{0}(t)\right)^{T} \in D\left(A^{0}\right)$ for all time, and $F=(f, g)^{T} \in H$. (iii).

Here again, we use Theorem 1.1, and we have to check (i), (ii), and

The proof of (i) is similar to what was done in Section 1.1, and we do not repeat it.

Let us now proceed with the proof of (ii):

Lemma 1.4. For every $U \in D\left(A^{0}\right),\left(A^{0} U, U\right)_{H} \geq 0$.

Proof. For $U \in D\left(A^{0}\right)$ we write:

$$
\begin{aligned}
\left(A^{0} U, U\right)_{H} & =\int_{0}^{L}\left[\left(\bar{U}_{0} u_{x}+\psi_{x}\right) u+\left(\bar{U}_{0} \lambda^{2} \psi_{x}+u_{x}\right) \psi\right] d x \\
& =\frac{1}{2} \bar{U}_{0}\left[u^{2}(L)-u^{2}(0)+\lambda^{2} \psi^{2}(L)-\lambda^{2} \psi^{2}(0)\right]+[u \psi]_{x=0}^{x=L}
\end{aligned}
$$


Thanks to the boundary conditions (1.28), we find:

$$
\left(A^{0} U, U\right)_{H}=\frac{1}{2} \bar{U}_{0} \lambda^{2} \psi^{2}(L)+\frac{1}{2} \bar{U}_{0} \lambda^{2}\left(1-\bar{U}_{0}^{2} \lambda^{2}\right) \psi^{2}(0) .
$$

Since $1 / \lambda>\bar{U}_{0}>0$, this last equation shows that $A$ is a positive operator, and ends the proof of Lemma 1.4.

To apply Theorem 1.1, we need one more result, proved in the following lemma:

Lemma 1.5. The operator $A^{0}+\mu I$ is onto, $\forall \mu>0$.

Proof. Let $\mu$ be a positive real, and let $(f, g) \in H$; we look for $\left(u^{0}, \psi^{0}\right) \in$ $D\left(A^{0}\right)$ such that:

$$
\left\{\begin{array}{l}
\bar{U}_{0} u_{x}^{0}+\psi_{x}^{0}+\mu u^{0}=f, \\
\bar{U}_{0} \psi_{x}^{0}+\frac{1}{\lambda^{2}} u_{x}^{0}+\mu \psi^{0}=g .
\end{array}\right.
$$

with:

$$
\left\{\begin{array}{l}
u^{0}(0)+\bar{U}_{0} \lambda^{2} \psi^{0}(0)=0 \\
u^{0}(L)=0
\end{array}\right.
$$

We need to integrate the system by elementary calculations. Classically, we first look for the general solution of the homogeneous system:

$$
\left\{\begin{array}{l}
\bar{U}_{0} u_{x}^{0}+\psi_{x}^{0}+\mu u^{0}=0 \\
\bar{U}_{0} \psi_{x}^{0}+\frac{1}{\lambda^{2}} u_{x}^{0}+\mu \psi^{0}=0 .
\end{array}\right.
$$

Looking for $\left(u^{0}, \psi^{0}\right)$ of the form $\left(A^{0}, B^{0}\right) e^{R^{0} x}$ we obtain:

$$
\left\{\begin{array}{l}
A^{0}\left(\bar{U}_{0} R^{0}+\mu\right)+R^{0} B^{0}=0, \\
B^{0}\left(\bar{U}_{0} R^{0}+\mu\right)+\frac{1}{\lambda^{2}} R^{0} A^{0}=0,
\end{array}\right.
$$

from which we infer the characteristic equation (for $R^{0}$ ):

$$
P^{0}\left(R^{0}\right)=\left(\bar{U}_{0} R^{0}+\mu\right)^{2}-\frac{R^{02}}{\lambda^{2}}=0
$$

The roots of the polynomial $P^{0}$ are:

$$
R_{1}^{0}=-\frac{\mu}{1 / \lambda+\bar{U}_{0}}<0, \text { and } R_{2}^{0}=\frac{\mu}{1 / \lambda-\bar{U}_{0}}>0 .
$$

Thus the general solution of problem (1.38) is of the form:

$$
\left(\begin{array}{c}
u^{0} \\
\psi^{0}
\end{array}\right)=\sum_{i=1}^{2}\left(\begin{array}{c}
A_{i}^{0} \\
B_{i}^{0}
\end{array}\right) e^{R_{i}^{0} x}
$$


In (1.38), $A_{i}^{0}$ is related to $B_{i}^{0}$ by $(1.39)$, that is

$$
B_{i}^{0}=b_{i}^{0} A_{i}^{0}
$$

where $b_{i}^{0}=\mu / R_{i}^{0}+\bar{U}_{0}$. Actually, $\left(b_{1}^{0}, b_{2}^{0}\right)=(-1 / \lambda, 1 / \lambda)$.

Hence, the general solution of (1.38) is:

$$
\left(\begin{array}{l}
u^{0} \\
\psi^{0}
\end{array}\right)=\sum_{i=1}^{2}\left(\begin{array}{c}
1 \\
b_{i}^{0}
\end{array}\right) A_{i}^{0} e^{R_{i}^{0} x}
$$

In view of using the Duhamel formula, we rewrite (1.36) in the form

$$
M Y^{\prime}(x)+\mu Y(x)=B(x)
$$

where:

$$
Y(x)=\left(\begin{array}{c}
u^{0} \\
\psi^{0}
\end{array}\right), M=\left(\begin{array}{cc}
\bar{U}_{0} & 1 \\
\frac{1}{\lambda^{2}} & \bar{U}_{0}
\end{array}\right), B(x)=\left(\begin{array}{c}
f(x) \\
g(x)
\end{array}\right) .
$$

The general solution of (1.38) is:

$$
Y=\sum_{i=1}^{2}\left(\begin{array}{c}
1 \\
b_{i}^{0}
\end{array}\right) A_{i}^{0} e^{R_{i}^{0} x}
$$

and we look for a solution of (1.36) of the form

$$
Y(x)=\sum_{i=1}^{2}\left(\begin{array}{c}
1 \\
b_{i}^{0}
\end{array}\right) A_{i}^{0}(x) e^{R_{i}^{0} x} .
$$

The matrix $M$ is invertible, since $\bar{U}_{0}^{2}-1 / \lambda^{2}<0$, thus, we should have:

$$
\sum_{i=1}^{2}\left(\begin{array}{c}
1 \\
b_{i}^{0}
\end{array}\right) A_{i}^{0^{\prime}}(x) e^{R_{i}^{0} x}=M^{-1} B(x)=: \tilde{B}(x) .
$$

Hence:

$$
\left\{\begin{aligned}
A_{1}^{0^{\prime}} e^{R_{1}^{0} x}+A_{2}^{0^{\prime}} e^{R_{2}^{0} x} & =\tilde{B}_{1}(x) \\
b_{1}^{0} A_{1}^{0^{\prime}} e^{R_{1}^{0} x}+b_{2}^{0} A_{2}^{0^{\prime}} e^{R_{2}^{0} x} & =\tilde{B}_{2}(x) .
\end{aligned}\right.
$$

System (1.50) is clearly regular since $b_{1}^{0} \neq b_{2}^{0}$, and from it we compute the $A_{i}^{0^{\prime}}$ using the Cramer's formula, and we then write:

$$
A_{i}^{0}(x)=\int_{0}^{x} A_{i}^{0^{\prime}}(u) d u+A_{i}^{0}(0), \quad i=1 . .2 .
$$

Since the $A_{i}^{0^{\prime}}$ were derived from (1.50), we only need to determine the coefficients $A_{i}^{0}(0)$, which we do using the boundary conditions (1.28). Hence we obtain the following system: 


$$
\left\{\begin{aligned}
A_{1}^{0}(0)\left(1+\lambda \bar{U}_{0}\right) & + & A_{2}^{0}(0)\left(1-\lambda \bar{U}_{0}\right) & =0, \\
A_{1}^{0}(0) e^{R_{1}^{0} L} & + & A_{2}^{0}(0) e^{R_{2}^{0} L} & =\lambda^{0}
\end{aligned}\right.
$$

where $\lambda^{0}=-\sum_{i=1}^{2}\left\{e^{R_{i}^{0} L} \int_{0}^{L} A_{i}^{0^{\prime}}(t) d t\right\}$.

This system is regular, we can uniquely determine the coefficients $A_{i}^{0}(0)$, and then obtain the exact expressions of the $A_{i}^{0}$, thanks to (1.51). We then have the solution of system (1.36) with (1.48); this shows that the operator $A^{0}+\mu I$ is onto.

We are now able to apply theorem (1.1) and we find:

Theorem 1.3. The problem (1.1)-(1.3) is well-posed, that is for every $(f, g) \in L^{1}(0, T ; H)$, and every $\left(u_{0}, \psi_{0}\right) \in D\left(A^{0}\right)$, there exists a unique solution $U^{0}=\left(u^{0}, \psi^{0}\right)$ of (1.27)-(1.29), with $U^{0} \in \mathcal{C}([0, T] ; H), d U^{0} / d t \in$ $L^{\infty}(0, T ; H)$.

\subsection{Convergence as $\varepsilon$ goes to 0 .}

We want to prove that, when $\varepsilon$ goes to zero, the solutions of (1.1)(1.3) do converge to those of (1.27)-(1.29). As numerically shown in [RTT04], some boundary layers appear at the boundary $x=0$. Actually we will prove this convergence by using and explicitly computing the correctors corresponding to these boundary layers..

The ansatz for the correctors $\left(\theta_{u}, \theta_{\psi}\right)$ is the following:

$$
\left(\begin{array}{c}
\theta_{u} \\
\theta_{\psi}
\end{array}\right)=\left(\begin{array}{c}
A \\
B
\end{array}\right) e^{-r x / \varepsilon}
$$

where $\theta_{u}, \theta_{\psi}$ satisfy

$$
\left\{\begin{aligned}
\bar{U}_{0} \theta_{u x}+\theta_{\psi x}-2 \varepsilon \theta_{u x x} & =0, \\
\bar{U}_{0} \theta_{\psi x}+\frac{1}{\lambda^{2}} \theta_{u x} & =0 .
\end{aligned}\right.
$$

and the boundary conditions

$$
\left\{\begin{array}{l}
\theta_{u}(0)=-u^{0}(0) \\
\theta_{\psi}(0)=-\psi^{0}(0) \\
\theta_{u}(L)=0
\end{array}\right.
$$


Hence we should have:

$$
\left\{\begin{aligned}
-\bar{U}_{0} \frac{r}{\varepsilon} A-\frac{r}{\varepsilon} B-2 \varepsilon \frac{r^{2}}{\varepsilon^{2}} A & =0 \\
-\bar{U}_{0} \frac{r}{\varepsilon} B-\frac{1}{\lambda^{2}} \frac{r}{\varepsilon} A & =0 .
\end{aligned}\right.
$$

The characteristic equation of system (1.54) reads

$$
r\left|\begin{array}{cc}
\bar{U}_{0}+2 r & 1 \\
\frac{1}{\lambda^{2}} & \bar{U}_{0}
\end{array}\right|=0,
$$

producing the solution $r=0$ and the solution

$$
r=\frac{1}{2 \bar{U}_{0} \lambda^{2}}-\frac{\bar{U}_{0}}{2}>0 .
$$

We can thus write $\theta_{u}$ and $\theta_{\psi}$ as follows:

$$
\left\{\begin{array}{l}
\theta_{u}=A e^{-r x / \varepsilon}+C \\
\theta_{\psi}=B e^{-r x / \varepsilon}+D
\end{array}\right.
$$

where $A$ and $B$ are linked by the second equation (1.54), so that:

$$
B=-\frac{1}{\bar{U}_{0} \lambda^{2}} A
$$

We then determine the coefficients $A, C, D$, using the boundary conditions (1.55) and we find

$$
\left\{\begin{aligned}
A+C & =-u^{0}(0), \\
B+D & =-\psi^{0}(0), \\
A e^{-r L / \varepsilon}+C & =0 .
\end{aligned}\right.
$$

After computation, we obtain:

$$
\left\{\begin{array}{l}
A=-\frac{1}{1-e^{-r L / \varepsilon}} u^{0}(0), \\
B=\frac{1}{\bar{U}_{0} \lambda^{2}\left(1-e^{-r L / \varepsilon}\right)} u^{0}(0), \\
C=\frac{e^{-r L / \varepsilon}}{1-e^{-r L / \varepsilon}} u^{0}(0), \\
D=-\frac{e^{-r L / \varepsilon}}{\bar{U}_{0} \lambda^{2}\left(1-e^{-r L / \varepsilon}\right)} u^{0}(0) .
\end{array}\right.
$$

Thus:

$$
\left\{\begin{array}{l}
\theta_{u}(x)=-u^{0}(0) e^{-r x / \varepsilon}+e_{u} \\
\theta_{\psi}(x)=-\psi^{0}(0) e^{-r x / \varepsilon}+e_{\psi} .
\end{array}\right.
$$

where $e_{u}$ and $e_{\psi}$ are some terms exponentially small in norm in all classical spaces. 
Having determined the correctors $\theta_{u}$ and $\theta_{\psi}$ we set $w_{u}=u-u^{0}-\theta_{u}$, $w_{\psi}=\psi-\psi^{0}-\theta_{\psi}$, where $\left(u^{\varepsilon}, \psi^{\varepsilon}\right)$ is solution of (1.1)-(1.3), and $\left(u^{0}, \psi^{0}\right)$ is solution of (1.27)-(1.29). We intend to prove the following result which concludes this study:

Theorem 1.4. We assume that $u_{x x}^{0} \in L^{\infty}\left(0, T ; L^{2}(0, L)\right)^{3}$. Then, as $\varepsilon \longrightarrow 0, w_{u}$ and $w_{\psi}$ are $O\left(\varepsilon^{1 / 2}\right)$ in $L^{\infty}\left(0, T ; L^{2}(0, L)\right)$.

Since $\theta_{u}$ and $\theta_{\psi}$ are $O\left(\varepsilon^{1 / 2}\right)$ in $L^{\infty}\left(L^{2}\right)$, we infer from the previous assumption that, as $\varepsilon \longrightarrow 0$,

$$
u^{\varepsilon} \longrightarrow u^{0}, \psi^{\varepsilon} \longrightarrow \psi^{0} \text { in } L^{\infty}\left(0, T ; L^{2}(0, L)\right) .
$$

Proof. It is clear that $w_{u}$ and $w_{\psi}$ verify:

$$
\left\{\begin{aligned}
w_{u t}+\bar{U}_{0} w_{u x}+w_{\psi x}-2 \varepsilon w_{u x x} & =2 \varepsilon u_{x x}^{0}-\theta_{u t} \\
w_{\psi t}+\bar{U}_{0} w_{\psi x}+\frac{1}{\lambda^{2}} w_{u x} & =-\theta_{\psi t} .
\end{aligned}\right.
$$

with

$$
w_{u}(0, t)=0, w_{\psi}(0, t)=0, w_{u}(L, t)=0,
$$

and

$$
w_{u}(x, 0)=0, w_{\psi}(x, 0)=0 .
$$

But

$$
\left\{\begin{array}{l}
\theta_{u}(x)=-u^{0}(0) e^{-r x / \varepsilon}+e_{u}=O\left(\varepsilon^{1 / 2}\right) \text { in } L^{\infty}\left(L^{2}\right) \\
\theta_{\psi}(x)=-\psi^{0}(0) e^{-r x / \varepsilon}+e_{\psi}=O\left(\varepsilon^{1 / 2}\right) \text { in } L^{\infty}\left(L^{2}\right)
\end{array}\right.
$$

Hence we have:

$$
\left\{\begin{aligned}
w_{u t}+\bar{U}_{0} w_{u x}+w_{\psi x}-2 \varepsilon w_{u x x} & =h_{u} \\
w_{\psi t}+\bar{U}_{0} w_{\psi x}+\frac{1}{\lambda^{2}} w_{u x} & =h_{\psi}
\end{aligned}\right.
$$

where $h_{u}$ and $h_{\psi}$ are the right-hand sides of the two equations (1.69), so that $\left\|h_{u}\right\|,\left\|h_{\psi}\right\|=O\left(\varepsilon^{1 / 2}\right)$ in $L^{\infty}\left(0, T ; L^{2}(0, L)\right)$.

Multiplying the first equation of (1.65) by $2 w_{u}$, and the second equation by $2 \lambda^{2} w_{\psi}$, summing and integrating, we obtain:

$$
\begin{aligned}
\frac{d}{d t} \int_{0}^{L}\left(w_{u}^{2}(x, t)+\lambda^{2} w_{\psi}^{2}(x, t)\right) d x=-\bar{U}_{0} w_{\psi}^{2}(L)-4 \varepsilon \int_{0}^{L} w_{u x}^{2}(x, t) d x \\
\quad-\int_{0}^{L}\left(w_{u}(x, t) w_{\psi x}(x, t)+w_{u x}(x, t) w_{\psi}(x, t)\right) d x \\
\quad+2 \int_{0}^{L}\left(h_{u}(x, t) w_{u}(x, t)+\lambda^{2} h_{\psi}(x, t) w_{\psi}(x, t)\right) d x
\end{aligned}
$$

\footnotetext{
${ }^{3}$ See Remark 1.4 hereafter.
} 
Dropping the negative terms, and using the inequality $2 a b \leq a^{2}+b^{2}$, we finally find:

$$
\frac{d}{d t} \int_{0}^{L}\left(w_{u}^{2}(x, t)+\lambda^{2} w_{\psi}^{2}(x, t)\right) d x \leq O(\varepsilon)+\int_{0}^{L}\left(w_{u}^{2}+\lambda^{2} w_{\psi}^{2}\right) d x
$$

from which we derive, thanks to the Gronwall lemma, that $w_{u}$ and $w_{\psi}$ are $O\left(\varepsilon^{1 / 2}\right)$ in $L^{\infty}\left(0, T ; L^{2}(0, L)\right)$.

Finally, we use (1.68) and the definitions of $\left(w_{u}, w_{\psi}\right)$ to achieve the proof of (1.64).

Remark 1.4. : Using the so called compatibility conditions (as in e.g [Tem82]), we can derive conditions on the initial data $u_{0}, \psi_{0}\left(\right.$ or $\left.\xi_{0}, \eta_{0}\right)$ which guarantee this regularity property on $u_{x x}^{0}$.

For instance, differentiating equations (1.27) in time, we obtain for $U_{t}^{0}=\left(u_{t}^{0}, \psi_{t}^{0}\right)$ an equation very similar to (1.27) for which a result similar to Theorem 1.3 applies. For that purpose we need to assume that $\left(f_{t}, g_{t}\right) \in L^{1}(0, T ; H)$ and that $\left.U_{t}^{0}\right|_{t}=0$ is in $D\left(A^{0}\right)$. In view of $(1.27),\left(u_{t}^{0}, \psi_{t}^{0}\right)$ belongs to $D\left(A^{0}\right)$ if, firstly, $\left.\left(u_{t x}^{0}, \psi_{t x}^{0}\right)\right|_{t=0}$ belongs to $L^{2}(0, L)^{2}$; differenciating equations $(1.27)$ in $x$, we see that this is true if $u_{0 x x}, \psi_{0 x x},\left.f_{x}\right|_{t=0},\left.g_{x}\right|_{t=0}$ are in $L^{2}(0, L)$. Furthermore we need the boundary conditions

$$
\left(u_{t}^{0}+\bar{U}_{0} \lambda^{2} \psi_{t}^{0}\right)(x=0, t=0)=u_{t}^{0}(x=L, t=0)=0,
$$

to be satisfied. In view of (1.27) written at $t=0$, we obtain the compatibility conditions for $u_{0}, \psi_{0}, f, g$ :

$$
\left\{\begin{array}{r}
2 \bar{U}_{0} u_{0 x}(0)+\left(1+\bar{U}_{0}^{2} \lambda^{2}\right) \psi_{0 x}(0)=f(0,0)+\bar{U}_{0} \lambda^{2} g(0,0), \\
\bar{U}_{0} u_{0 x}(L)+\psi_{0 x}(L)=f(L, 0) .
\end{array}\right.
$$

Hence, if conditions (1.72) are satisfied, as well as the regularity conditions above and below $\left(f_{t}, g_{t} \in L^{1}\left(0, T ; L^{2}(0, L)\right), u_{0 x x}, \psi_{0 x x},\left.f_{x}\right|_{t=0},\left.g_{x}\right|_{t=0}\right.$ are in $\left.L^{2}(0, L)\right)$, then, as for Theorem 1.3, the Hille-Yosida theorem gives the existence and uniqueness of $U_{t}^{0}=\left(u_{t}^{0}, \psi_{t}^{0}\right)$ in $\mathcal{C}([0, T] ; H)$, with $U_{t t}^{0}=\left(u_{t t}^{0}, \psi_{t t}^{0}\right)$ in $L^{\infty}(0, T ; H)$. Therefore $u_{0 x t}, \psi_{0 x t}$ are in $L^{\infty}\left(0, T ; L^{2}(0, L)\right)$. Differenciating equations (1.27) in $x$ and assuming also that $f_{x}, g_{x}$ are in $L^{\infty}\left(0, T ; L^{2}(0, L)\right)$, we conclude that $u_{x x}^{0}$ and $\psi_{x x}^{0}$ are in $L^{\infty}\left(0, T ; L^{2}(0, L)\right)$.

Remark 1.5. : This article is devoted to the subcritical case i.e when $\lambda^{-1}>\bar{U}_{0}>0$ and $\beta>0$ in (1.31). This case is more difficult than the supercritical case corresponding to $\bar{U}_{0}>\lambda^{-1}>0$ so that $\beta<0$ in (1.31). In the supercritical case both characteristics enter the domain 
on the left-hand side, and the natural boundary conditions for (1.31) read

$$
\xi^{0}(0, t)=\eta^{0}(0, t)=0,
$$

or in the $u^{0}, \psi^{0}$ variables,

$$
u^{0}(0, t)=\psi^{0}(0, t)=0 .
$$

With exactly the same methods as for Theorem 1.4, one can prove that $\left(u^{\varepsilon}, \psi^{\varepsilon}\right)$ converges to $\left(u^{0}, \psi^{0}\right)$, where $u^{0}, \psi^{0}$, are now solutions of $(1.27),(1.74)$ and (1.34) (and a similar result for $\xi^{\varepsilon}, \eta^{\varepsilon}$ ). In that case there is no reflection of wave at the right boundary $x=L$ : the natural boundary conditions for the system $\left(\xi^{0}, \eta^{0}\right)$ or $\left(u^{0}, \psi^{0}\right)$ are already "transparent", and there is no need at all for the developments to come in Section 2.

For further explanations the reader is refered to classical references on hyperbolic systems, such as e.g. [Lax86, Ser96].

\section{TRANSPARENT BOUNDARY CONDITIONS}

As shown in [RTT04] and as evidenced by the first condition of (1.28), the system (1.1) supplemented with the boundary conditions (1.2) produces, in the limit $\varepsilon \longrightarrow 0$, some reflection phenomena at the boundary. These reflections are not desirable in the context in which these equations were introduced (see the discussion in [RTT04]). For that reason, in this section and in the next one, we propose to supplement the equations (1.1) with a different set of boundary conditions of transparent type (see e.g. [EM77],[TH86]). With such conditions, the solution of (1.1) converge - when $\varepsilon$ goes to zero - to a solution of the limit system (1.27), supplemented with boundary conditions which do not produce reflections at the boundary and which are in some sense, the "natural" boundary conditions for (1.27).

\subsection{The limit system with natural boundary conditions.}

In order to better understand the limit system (1.27), we use its diagonal form (1.31). We recall that $1 / \lambda>\bar{U}_{0}$ so that $\alpha>\beta>0$. In the $\xi, \eta$ variables, the system (1.1) becomes:

$$
\left\{\begin{array}{l}
\xi_{t}^{\varepsilon}+\alpha \xi_{x}^{\varepsilon}-\varepsilon\left(\xi^{\varepsilon}+\eta^{\varepsilon}\right)_{x x}=f+\lambda g, \\
\eta_{t}^{\varepsilon}-\beta \eta_{x}^{\varepsilon}-\varepsilon\left(\xi^{\varepsilon}+\eta^{\varepsilon}\right)_{x x}=f-\lambda g,
\end{array} \quad 0<x<L, \quad t>0 .\right.
$$


Setting formally $\varepsilon=0$ in (2.1), we obtain the above equations (1.31). These two equations are uncoupled, they are simple transport equations, with waves propagating at speed $\alpha$ for $\xi$ and $-\beta$ for $\eta$. The most "natural" boundary conditions for (1.31) are conditions of the type

$$
\xi^{0}(0, t)=\xi_{l}(t), \eta^{0}(L, t)=\eta_{r}(t), \quad t>0 .
$$

Of course, the problem (1.31)-(2.2) with initial data (1.33) is wellposed, and its well-known solution, in the homogeneous case $f=g=0$, $\xi_{l}=\eta_{r}=0$, is the pair of travelling waves $^{4}$

$$
\left\{\begin{array}{l}
\xi^{0}(x, t)=\xi_{0}(x-\alpha t) \\
\eta^{0}(x, t)=\eta_{0}(x+\beta t) .
\end{array}\right.
$$

Back to the $(u, \psi)$ variables, the homogeneous problem is:

$$
\left\{\begin{array}{l}
u_{t}^{0}+\bar{U}_{0} u_{x}^{0}+\psi_{x}^{0}=0, \\
\psi_{t}^{0}+\bar{U}_{0} \psi_{x}^{0}+\frac{1}{\lambda^{2}} u_{x}^{0}=0, \quad 0<x<L, \quad t>0
\end{array}\right.
$$

with the boundary conditions:

$$
\left\{\begin{array}{l}
u^{0}(0, t)+\lambda \psi^{0}(0, t)=0, \\
u^{0}(L, t)-\lambda \psi^{0}(L, t)=0,
\end{array} \quad t>0\right.
$$

Its solution reads, thanks to $(2.3)$ :

$$
\begin{aligned}
u^{0}(x, t) & =\frac{1}{2}\left[\left(u_{0}+\lambda \psi_{0}\right)(x-\alpha t)+\left(u_{0}-\lambda \psi_{0}\right)(x+\beta t)\right], \\
\psi^{0}(x, t) & =\frac{1}{2 \lambda}\left[\left(u_{0}+\lambda \psi_{0}\right)(x-\alpha t)-\left(u_{0}-\lambda \psi_{0}\right)(x+\beta t)\right] .
\end{aligned}
$$

Naturally, in the nonhomogeneous case, $\left(u^{0}, \psi^{0}\right)$ are also well-defined, but their expression are slightly more complicated.

From now on, we restrict ourselves to the homogeneous boundary conditions $\xi_{l}=\eta_{r}=0$, and we assume that $\left(u^{0}, \psi^{0}\right)$ are given, computed by (2.3). The aim of the following section is to propose and study a set of boundary conditions supplementing (1.1) and such that its solution converges, as $\varepsilon$ goes to zero, to the "natural" solution given in (2.3). These boundary conditions are called transparent boundary conditions $(\mathrm{TBC})^{5}$.

\footnotetext{
${ }^{4}$ Here we extend the definition of the initial data to all of $\mathbb{R}$ by setting: $\xi_{0}(x), \eta_{0}(x)=0$ if $x \notin[0, L]$.

${ }^{5}$ For the nonspecialist we recall that this expression comes from the fact that there is no reflection of waves at the boundary so that waves go through the boundary, as if it were not there. This is an essential feature for the problem which motivated this study, namely numerical predictions (or simulations) in a
} 
2.2. The transparent boundary conditions. We consider the following equations, in the $(u, \psi)$ variables:

$$
\left\{\begin{array}{l}
u_{t}^{\varepsilon}+\bar{U}_{0} u_{x}^{\varepsilon}+\psi_{x}^{\varepsilon}-2 \varepsilon u_{x x}^{\varepsilon}=f, \\
\psi_{t}^{\varepsilon}+\bar{U}_{0} \psi_{x}^{\varepsilon}+\frac{1}{\lambda^{2}} u_{x}^{\varepsilon}=g,
\end{array} \quad 0<x<L, t>0,\right.
$$

with initial data

$$
\left\{\begin{array}{l}
u^{\varepsilon}(x, 0)=u_{0}(x), \\
\psi^{\varepsilon}(x, 0)=\psi_{0}(x),
\end{array} \quad 0<x<L .\right.
$$

We supplement these equations with the following boundary conditions:

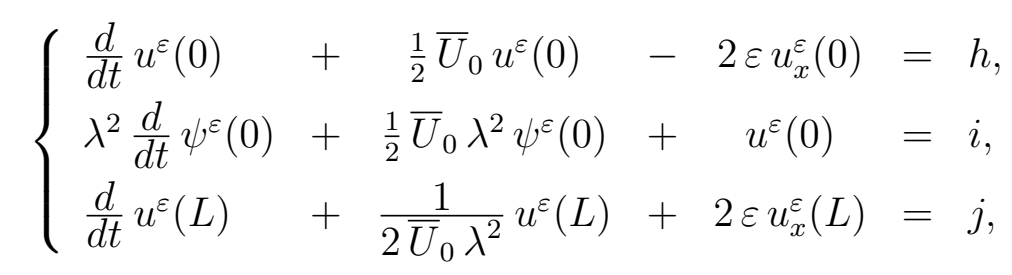

where $h, i, j$ are the following functions of $t$ (through $u^{0}$ and $\psi^{0}$ ):

$$
\left\{\begin{aligned}
h & =\frac{d}{d t} u^{0}(0) \quad+\frac{1}{2} \bar{U}_{0} u^{0}(0), \\
i & =\lambda^{2} \frac{d}{d t} \psi^{0}(0)+\frac{1}{2} \bar{U}_{0} \lambda^{2} \psi^{0}(0)+u^{0}(0), \\
j & =\frac{d}{d t} u^{0}(L)+\frac{1}{2 \bar{U}_{0} \lambda^{2}} u^{0}(L) .
\end{aligned}\right.
$$

Remark 2.1. : Since $(u, \psi) \in D(A),(u, \psi) \in H^{2} \times H^{1} \subset \mathcal{C}^{1} \times \mathcal{C}^{0}$, and the quantities in (2.9) are well defined.

Remark 2.2. : As in Section 1, from now on we drop the superscript $\varepsilon$ and write $(u, \psi)$ instead of $\left(u^{\varepsilon}, \psi^{\varepsilon}\right)$.

We want to prove the well-posedness of problem (2.7)-(2.9), and the convergence of its solutions to the solution $\left(u^{0}, \psi^{0}\right)$ of $(2.6)$ as $\varepsilon$ goes to zero. For the well-posedness we will use the Hille-Yosida theorem

limited domain with artificial (nonnatural) boundaries. The expressions "transparent" and "nonreflecting" boundary conditions are both used in the literature [Str77, RS80, RS81, Hal91, Tou97]. 
again, in the following functional framework:

$$
\begin{aligned}
& H=L^{2}(0, L) \times L^{2}(0, L) \times \mathbb{R}^{3}, \\
& D(A)=\left\{U=\left(U_{j}\right)_{1 \leq j \leq 5} \in H,\left(U_{1}, U_{2}\right)=(u, \psi) \in H^{2} \times H^{1},\right. \\
& \left.U_{3}=u(0), U_{4}=\psi(0), U_{5}=u(L)\right\}, \\
& A\left(\begin{array}{l}
u \\
\psi \\
u(0) \\
\psi(0) \\
u(L)
\end{array}\right)=\left(\begin{array}{l}
\bar{U}_{0} u_{x}+\psi_{x}-2 \varepsilon u_{x x} \\
\bar{U}_{0} \psi_{x}+\frac{1}{\lambda^{2}} u_{x} \\
\frac{1}{2} \bar{U}_{0} u(0)-2 \varepsilon u_{x}(0) \\
\frac{1}{2} \bar{U}_{0} \lambda^{2} \psi(0)+u(0) \\
\frac{1}{2 \bar{U}_{0} \lambda^{2}} u(L)+2 \varepsilon u_{x}(L)
\end{array}\right), \forall\left(\begin{array}{l}
u \\
\psi \\
u(0) \\
\psi(0) \\
u(L)
\end{array}\right) \in D(A) .
\end{aligned}
$$

We endow $H$ with the following inner product:

$$
\left.(U, V)_{H}=\int_{0}^{L}\left\{U_{1} V_{1}\right)+\lambda^{2} U_{2} V_{2}\right\} d x+U_{3} V_{3}+U_{4} V_{4}+U_{5} V_{5}
$$

Equations (2.7)-(2.9) can now be written in the functional form

$$
\frac{d U}{d t}+A U=F, U(0)=U_{0}
$$

where

$$
\begin{aligned}
& U_{0}=\left(u_{0}, \psi_{0}, u_{0}(0), \psi_{0}(0), u_{0}(L)\right)^{T} \in D(A), \\
& U(t)=(u(t), \psi(t), u(0, t), \psi(0, t), u(L, t))^{T} \in D(A) \text { for all time, }
\end{aligned}
$$

and $\quad F=(f, g, h, i, j)^{T} \in L^{1}(0, T ; H)$.

In order to apply Theorem 1.1 we have to check its hypotheses (i), (ii), and (iii). We skip the technical proof of (i), done as in Lemma 1.1, and in the following Lemmas 2.1 and 2.2, we check the two other hypotheses of the Hille-Yosida theorem.

Lemma 2.1. For every $U \in D(A),(A U, U)_{H} \geq 0$. 
Proof. For $U \in D(A)$, we compute the inner product

$$
\begin{aligned}
(A U, U)_{H}= & \frac{1}{2} \bar{U}_{0} u^{2}(L)-\frac{1}{2} \bar{U}_{0} u^{2}(0)+u(L) \psi(L)-u(0) \psi(0) \\
& +\frac{1}{2} \bar{U}_{0} \lambda^{2} \psi^{2}(L)-\frac{1}{2} \bar{U}_{0} \lambda^{2} \psi^{2}(0)-2 \varepsilon\left[u_{x} u\right]_{0}^{L} \\
& +2 \varepsilon\left|u_{x}\right|_{L^{2}}^{2} \\
& +\frac{1}{2} \bar{U}_{0} u^{2}(0)-2 \varepsilon u_{x}(0) u_{(}(0) \\
& +\frac{1}{2} \bar{U}_{0} \lambda^{2} \psi^{2}(0)+u(0) \psi(0) \\
& \left.+\frac{1}{2 \bar{U}_{0} \lambda^{2}} u^{2}(L)+2 \varepsilon u_{x}(L) u_{(} L\right)
\end{aligned}
$$

Dropping terms that cancel each other, we find:

$$
(A U, U)_{H}=2 \varepsilon\left|u_{x}\right|_{L^{2}}^{2}+\frac{1}{2} \bar{U}_{0} u^{2}(L)+\frac{1}{2 \bar{U}_{0} \lambda^{2}}\left(u(L)+\bar{U}_{0} \lambda^{2} \psi(L)\right)^{2} .
$$

This quantity is nonnegative, and the lemma is proven.

The last step is the following:

Lemma 2.2. The operator $A+\mu I$ is onto, $\forall \mu>0$.

Proof. Let $\mu$ be a positive real, and let $F=(f, g, h, j, k) \in H$.

We look for $(u, \psi, u(0), \psi(0), u(L)) \in D(A)$ such that:

$$
\left\{\begin{aligned}
\bar{U}_{0} u_{x}+\psi_{x}-2 \varepsilon u_{x x}+\mu u & =f, \\
\bar{U}_{0} \psi_{x}+\frac{1}{\lambda^{2}} u_{x}+\mu \psi & =g, \\
\frac{1}{2} \bar{U}_{0} u^{\varepsilon}(0)-2 \varepsilon u_{x}^{\varepsilon}(0)+\mu u(0) & =h, \\
\frac{1}{2} \bar{U}_{0} \lambda^{2} \psi^{\varepsilon}(0)+u^{\varepsilon}(0)+\mu \psi(0) & =j, \\
\frac{1}{2 \bar{U}_{0} \lambda^{2}} u^{\varepsilon}(L)+2 \varepsilon u_{x}^{\varepsilon}(L)+\mu u(L) & =k .
\end{aligned}\right.
$$

We intend to reduce this system to a variational equation for $u$ and use Lax-Milgram theorem for its solution. Before that, we express $\psi$ as a function of $u$.

Thanks to (2.12b), we have:

$$
\psi_{x}+\frac{\mu}{\bar{U}_{0}} \psi=\frac{g}{\bar{U}_{0}}-\frac{1}{\bar{U}_{0} \lambda^{2}} u_{x} .
$$


We integrate this equation and find:

$$
\psi=\Psi_{0}+\Psi_{1}(u)
$$

where

$$
\begin{aligned}
& \Psi_{0}=\frac{2}{2 \mu+\bar{U}_{0} \lambda^{2}} j e^{-\mu x / \bar{U}_{0}}+\frac{1}{\bar{U}_{0}} \int_{0}^{x} g\left(x^{\prime}\right) e^{-\mu\left(x-x^{\prime}\right) / \bar{U}_{0}} d x^{\prime} \\
& \Psi_{1}(u)=\frac{-2 u(0) e^{-\mu x / \bar{U}_{0}}}{2 \mu+\bar{U}_{0} \lambda^{2}}-\frac{1}{\bar{U}_{0} \lambda^{2}} \int_{0}^{x} u_{x}\left(x^{\prime}\right) e^{-\mu\left(x-x^{\prime}\right) / \bar{U}_{0}} d x^{\prime}
\end{aligned}
$$

From (2.15) and (2.16), we see that $\Psi_{1}$ is a linear mapping from $H^{1}$ into itself, and that $\Psi_{0} \in H^{1}$. We thus have the following problem in $u$ :

$$
\left\{\begin{aligned}
\bar{U}_{0} u_{x}+\frac{d}{d x} \Psi_{1}(u)-2 \varepsilon u_{x x}+\mu u & =f-\frac{d}{d x} \Psi_{0}, \\
\frac{1}{2} \bar{U}_{0} u^{\varepsilon}(0)-2 \varepsilon u_{x}^{\varepsilon}(0)+\mu u(0) & =h \\
\frac{1}{2 \bar{U}_{0} \lambda^{2}} u^{\varepsilon}(L)+2 \varepsilon u_{x}^{\varepsilon}(L)+\mu u(L) & =k
\end{aligned}\right.
$$

The variational formulation of (2.17) reads: To find $u \in H^{1}(0, L)$, such that

$$
a(u, \tilde{u})=l(\tilde{u}), \forall \tilde{u} \in H^{1}(0, L),
$$

where

$$
\begin{aligned}
a(u, \tilde{u})= & \bar{U}_{0}\left(u_{x}, \tilde{u}\right)_{L^{2}}+\left(\frac{d}{d x} \Psi_{1}(u), \tilde{u}\right)_{L^{2}}+2 \varepsilon\left(u_{x}, \tilde{u}_{x}\right)_{L^{2}}+\mu(u, \tilde{u})_{L^{2}} \\
& +\left(\frac{1}{2} \bar{U}_{0}+\mu\right) u(0) \tilde{u}(0)+\left(\frac{1}{2 \bar{U}_{0} \lambda^{2}}+\mu\right) u(L) \tilde{u}(L), \\
l(\tilde{u})= & \left(f-\frac{d}{d x} \Psi_{0}, \tilde{u}\right)+h \tilde{u}(0)+k \tilde{u}(L) .
\end{aligned}
$$

It is clear that $a$ is defined and bilinear continuous on $H^{1}(0, L)$ and that $l$ is defined and linear continuous on that space. We ought to prove the coercivity of $a$ on $H^{1}(0, L)$, and it is sufficient to establish it for $u$ belonging to a dense subspace of $H^{1}(0, L)$, say $\mathcal{C}^{2}([0, L])$. For such a $u$ we compute $a(u, u)$ :

$$
\begin{aligned}
a(u, u)= & \frac{1}{2} \bar{U}_{0} u^{2}(L)-\frac{1}{2} \bar{U}_{0} u^{2}(0)+\left(\frac{d}{d x} \Psi_{1}(u), u\right)+2 \varepsilon\left|u_{x}\right|_{L^{2}}^{2} \\
& +\left(\frac{1}{2} \bar{U}_{0}+\mu\right) u^{2}(0)+\left(\frac{1}{2 \bar{U}_{0} \lambda^{2}}+\mu\right) u^{2}(L)+\mu|u|_{L^{2}}^{2} .
\end{aligned}
$$


We know that $\Psi_{1}=\Psi_{1}(u)$ is solution of:

$$
\left\{\begin{array}{l}
\frac{\partial \Psi_{1}}{\partial x}+\frac{\mu}{\bar{U}_{0}} \Psi_{1}=\frac{-1}{\bar{U}_{0} \lambda^{2}} u_{x} \\
\Psi_{1}(0)=\frac{-2}{2 \mu+\bar{U}_{0} \lambda^{2}} u(0) .
\end{array}\right.
$$

Hence we find:

$$
\begin{aligned}
a(u, u) & =\mu|u|_{L^{2}}^{2}+2 \varepsilon\left|u_{x}\right|_{L^{2}}^{2}+\frac{1}{2} \bar{U}_{0} u^{2}(L)+\mu\left(u^{2}(0)+u^{2}(L)\right) \\
+ & {\left[\Psi_{1} u\right]_{0}^{L}-\left(\Psi_{1}, \bar{U}_{0} \lambda^{2} \frac{d}{d x} \Psi_{1}+\mu \lambda^{2} \Psi_{1}\right)+\frac{1}{2 \bar{U}_{0} \lambda^{2}} u^{2}(L) . }
\end{aligned}
$$

Finally, using the boundary condition (2.20b)i we obtain the coercivity:

$$
a(u, u) \geq 2 \varepsilon\left|u_{x}\right|_{L^{2}}^{2}+\mu|u|_{L^{2}}^{2}
$$

Hence we can use the Lax-Milgram theorem and we obtain the existence and uniqueness of a solution $u$ for (2.17). From (2.14), we see that $\psi \in H^{1}(0, L)$, and finally $u \in H^{2}(0, L)$, thanks to (2.12a). Hence $U=(u, \psi, u(0), \psi(0), u(L)) \in D(A)$, and $U$ is solution of $A U+\mu U=$ $F$. The lemma is proven.

To conclude this section, we can now write the following result:

Theorem 2.1. The problem (2.7)-(2.9) is well-posed, that is for every $(f, g) \in H$, and every $\left(u_{0}, \psi_{0}\right) \in D(A)$, there exists a unique solution $U=(u, \psi)$ of (2.7)-(2.9), with $U \in \mathcal{C}([0, T] ; H), d U / d t \in L^{\infty}(0, T ; H)$.

We next prove the convergence, when $\varepsilon$ goes to zero, of the solution of (2.7)-(2.9) to the solution (2.6) of the limit problem.

\subsection{Convergence for the transparent boundary conditions.}

We are here interested in two systems of equations. The first system is (1.27), supplemented with the boundary condition (2.5). The solutions of this system is given by (2.6). The second perturbed system of equations is (1.1) with boundary conditions (2.9).

Thanks to the previous results, we know that these two problems are well-posed. Our aim is now to prove that $\left(u^{\varepsilon}, \psi^{\varepsilon}\right)$, solution of (1.1), (2.9), converges to $\left(u^{0}, \psi^{0}\right)$, solution of $(1.27),(2.5)$, as $\varepsilon \longrightarrow 0$.

We set

$$
\tilde{u}=u^{\varepsilon}-u^{0}, \tilde{\psi}=\psi^{\varepsilon}-\psi^{0}
$$

and prove the following: 
Theorem 2.2. As $\varepsilon \longrightarrow 0, \tilde{u}$ and $\tilde{\psi}$ are $O\left(\varepsilon^{1 / 2}\right)$ in $L^{\infty}\left(0, T ; L^{2}(0, L)\right)$.

Proof. The equations for $(\tilde{u}, \tilde{\psi})$ read:

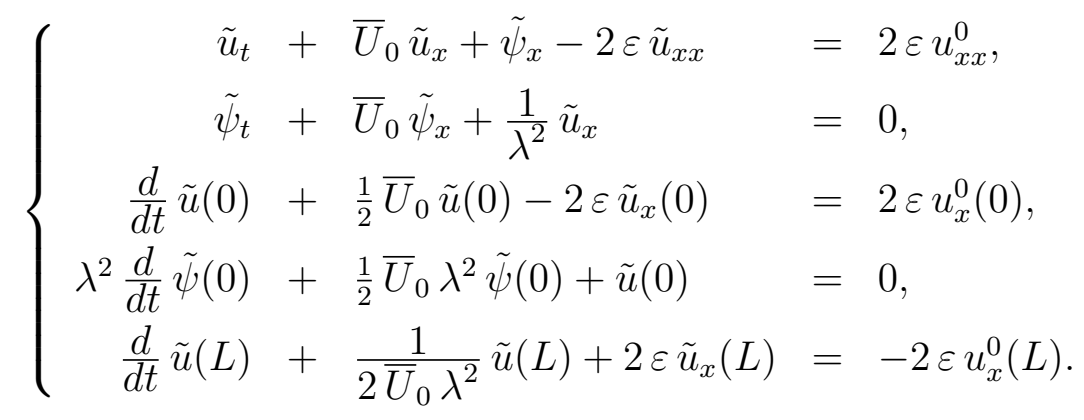

Taking the scalar product of each side of equation (2.23) with the corresponding component of $\tilde{U}$, we find after simplification ${ }^{6}$ :

$$
\begin{gathered}
\frac{1}{2} \frac{d}{d t} E(t)+2 \varepsilon\left|\tilde{u}_{x}\right|_{L^{2}}^{2}+\frac{1}{2} \bar{U}_{0} \lambda^{2}\left(\tilde{\psi}(L)+\frac{1}{\bar{U}_{0} \lambda^{2}} \tilde{u}(L)\right)^{2}+\frac{1}{2} \bar{U}_{0} \tilde{u}^{2}(L) \\
=2 \varepsilon\left\{\int_{0}^{L} u_{x x}^{0} \tilde{u} d x+u_{x}^{0}(0) \tilde{u}(0)-u_{x}^{0}(L) \tilde{u}(L)\right\},
\end{gathered}
$$

where $E(t)=\int_{0}^{L}\left(\tilde{u}^{2}+\lambda^{2} \tilde{\psi}^{2}\right) d x+\tilde{u}^{2}(0)+\tilde{\psi}^{2}(0)+\tilde{u}^{2}(L)$.

Hence, using the Cauchy-Schwarz inequality, we finally obtain:

$$
\frac{1}{2} \frac{d}{d t} E(t)+2 \varepsilon\left|\tilde{u}_{x}\right|_{L^{2}}^{2} \leq E(t)+K_{1} \varepsilon^{2},
$$

where $K_{1}$ depends only on $\left(u^{0}, \psi^{0}\right)$.

Using the Gronwall lemma, we conclude the proof of Theorem 2.2.

Remark 2.3. : We notice that for the purpose of this proof, we did not explicitly use the boundary conditions (2.5), and only used that $\left(u^{0}, \psi^{0}\right)$ are defined and satisfy (1.27). Hence we could also use $\left(u^{0}, \psi^{0}\right)$ from the boundary conditions (1.28) from Section 1, and still obtain convergence. Doing so, we would find a numerical solution, with $\varepsilon>0$ and these TBC, that produces some reflections. In other words, the boundary conditions are actually transparent only if $\left(u^{0}, \psi^{0}\right)$ are well chosen!

\footnotetext{
${ }^{6}$ Numerous terms cancel each other, which is one of the reasons for the choice of equations $(2.9)$
} 


\section{Another set of TBC}

The solution of the perturbed system (1.1),(2.9), necessitates the knowledge of the solution $\left(u^{0}, \psi^{0}\right)$ of the limit system $(1.27)$, which is easy to find in this case. In cases where the limit system is not easily accessible, the procedure above may not be practical. In fact in our computations described in [RTT04], we used a different set of perturbed equations which does not necessitate the knowledge of the limit solution. We now describe this system in its discrete form, in the case of homogeneous boundary conditions. We have not been able to show that, in the limit $\Delta x \longrightarrow 0$, this system is well-posed and, in fact, a naive count of the number of equations indicates that the continuous system may be overdetermined if no precautions are taken. However, as we said, this perturbed system has some computational advantages, and for $\Delta x>0$ fixed it is well-posed, the limit system is well-posed (but this is standard), and the perturbed system does converge to the expected limit when $\varepsilon \longrightarrow 0$ ( $\Delta x$ fixed).

We now give, for the sake of completeness, a brief description of these results which do not raise any essential difficulty, the systems being finite dimensional.

\subsection{Existence of a discrete solution.}

Let $N$ be a given integer. We set $h=L /(N+1)$. We want to compute the step functions

$$
\left\{\begin{array}{rl}
\xi_{h}(x, t) & =\sum_{j=0}^{N} \xi_{j}(t) w_{j}^{h}(x), \\
\eta_{h}(x, t) & =\sum_{j=0}^{N} \eta_{j}(t) w_{j}^{h}(x),
\end{array} \quad \forall t>0,\right.
$$

where $\left(\xi_{j}, \eta_{j}\right)(t)$ are unknown, and where $w_{j}^{h}=\chi_{[j h,(j+1) h)}$.

Similarly, we introduce $f_{h}$ and $g_{h}$ of the form

$$
\begin{cases}f_{h}(x, t)=\sum_{j=0}^{N} f_{j}(t) w_{j}^{h}(x), & \\ g_{h}(x, t)=\sum_{j=0}^{N} g_{j}(t) w_{j}^{h}(x), & \forall t>0,\end{cases}
$$


where $f_{j}(t)=f(j h, t)$ and $g_{j}(t)=g(j h, t)$.

We define the following discrete operators $\nabla_{h}$ and $\bar{\nabla}_{h}$ :

$$
\left\{\begin{array}{l}
\left(\nabla_{h} \cdot \varphi\right)_{j}=\frac{\varphi_{j+1}-\varphi_{j}}{h}, \forall j=0 . . N-1, \\
\left(\bar{\nabla}_{h} \cdot \varphi\right)_{j}=\frac{\varphi_{j}-\varphi_{j-1}}{h}, \forall j=1 . . N .
\end{array}\right.
$$

To be consistent with (2.2) in the homogeneous case, we set

$$
\xi_{0}(t)=0, \eta_{N}(t)=0, \forall t>0 .
$$

The discrete equations, derived from (3.1) for all $t>0$, read for $j=1, \ldots, N-1$ :

$$
\left\{\begin{array}{l}
\frac{d \xi_{j}}{d t}(t)+\alpha(\bar{\nabla} \xi)_{j}(t)-\varepsilon[\nabla \bar{\nabla}(\xi+\eta)]_{j}(t)=f_{j}(t) \\
\frac{d \eta_{j}}{d t}(t)-\beta(\nabla \eta)_{j}(t)-\varepsilon[\nabla \bar{\nabla}(\xi+\eta)]_{j}(t)=g_{j}(t)
\end{array}\right.
$$

We choose to supplement this system with the discrete boundary conditions

$$
\left\{\begin{aligned}
\frac{d \xi_{N}}{d t}(t)+\frac{\alpha}{h}\left(\xi_{N}-\xi_{N-1}-\eta_{N-1}\right)(t) & =f_{N}(t)-\frac{\alpha}{\beta} g_{N}(t), \\
\frac{d \eta_{0}}{d t}(t)-\frac{\beta}{h}\left(\xi_{1}+\eta_{1}-\eta_{0}\right)(t) & =g_{0}(t)-\frac{\beta}{\alpha} f_{0}(t) .
\end{aligned}\right.
$$

We consider the space $H=\mathbb{R}^{N-1} \times \mathbb{R}^{N-1} \times \mathbb{R} \times \mathbb{R} \simeq \mathbb{R}^{2 N}$. The domain of the operator, usually denoted $D\left(A_{h}\right)$, is all of $H$ since our operator $A_{h}$ is bounded. Each element $U_{h}$ in $H$ is of the form $U_{h}=\left(\xi_{1}, \ldots, \xi_{N-1}, \eta_{1}, \ldots, \eta_{N-1}, \xi_{N}, \eta_{0}\right)$.

For every $U_{h}$ in $H$, we set

$$
A_{h} U_{h}=\left(\begin{array}{rl}
\alpha(\bar{\nabla} \xi)_{j}-\varepsilon[\nabla \bar{\nabla}(\xi+\eta)]_{j}, & j=1 . . N-1 \\
-\beta(\nabla \eta)_{j}-\varepsilon[\nabla \bar{\nabla}(\xi+\eta)]_{j}, & j=1 . . N-1 \\
\frac{\alpha}{h}\left(\xi_{N}-\xi_{N-1}-\eta_{N-1}\right) & \\
-\frac{\beta}{h}\left(\xi_{1}+\eta_{1}-\eta_{0}\right) &
\end{array}\right) .
$$

Hence we can write (3.5) in the form:

$$
\frac{d U_{h}}{d t}+A_{h} U_{h}=F_{h}, \quad U_{h}(0)=U_{0, h},
$$

where:

$$
\begin{aligned}
U_{h} & =\left(\xi_{1}, \ldots, \xi_{N-1}, \eta_{1}, \ldots, \eta_{N-1}, \xi_{N}, \eta_{0}\right)^{T} \\
F_{h} & =\left(f_{j}, g_{j}, f_{N}-\frac{\alpha}{\beta} g_{N}, g_{0}-\frac{\beta}{\alpha} f_{0}\right)^{T}
\end{aligned}
$$


and $U_{0, h}$ is a spatial discretization of the initial data $U_{0}=\left(u_{0}, \psi_{0}\right)$. We notice the following theorem which is easy since (3.8) is a linear ordinary differential system with as many equations as unknowns.

Theorem 3.1. The problem (3.4)-(3.6) is well-posed, that is for every $(f, g) \in L^{1}(0, T ; H)$, and every $U_{0, h} \in H$, there exists a unique solution $U_{h}$ of (3.4)-(3.6), with $U_{h} \in \mathcal{C}([0, T] ; H), d U_{h} / d t \in L^{1}(0, T ; H)$.

We then consider the limit system obtained by setting formally $\varepsilon=0$ in (3.5), that is

$$
\begin{cases}\frac{d \xi_{j}^{0}}{d t}(t)+\alpha\left(\bar{\nabla} \xi^{0}\right)_{j}(t)=f_{j}(t), & 1 \leq j \leq N, t>0, \\ \frac{d \eta_{j}^{0}}{d t}(t)-\beta\left(\nabla \xi^{0}\right)_{j}(t)=g_{j}(t), & 0 \leq j \leq N-1, \quad t>0 .\end{cases}
$$

We define $\xi_{h}^{0}$ and $\eta_{h}^{0}$ as in (3.1), and impose the boundary conditions

$$
\xi_{0}^{0}=0, \eta_{N}^{0}=0,
$$

For the functional setting, the Hilbert space $H^{0}$ is $\mathbb{R}^{2 N}$, and for every $U=\left(\xi_{1}, \ldots, \xi_{N-1}, \eta_{1}, \ldots, \eta_{N-1}, \xi_{N}, \eta_{0}\right) \in H^{0}$ we write

$$
A_{h}^{0} U=\left(\begin{array}{rl}
\alpha(\bar{\nabla} \xi)_{j}, & j=1 . . N \\
-\beta(\nabla \eta)_{j}, & j=0 . . N-1
\end{array}\right) .
$$

The initial value problem is written as

$$
\frac{d U_{h}^{0}}{d t}+A_{h}^{0} U_{h}^{0}=F_{h}, \quad U_{h}^{0}(0)=U_{0, h} .
$$

This is again a linear differential system with as many equations as unknowns, so that the analogue of Theorem 3.1 is also easy:

Theorem 3.2. The problem (3.10)-(3.11) is well-posed, that is for every $(f, g) \in L^{1}\left(0, T ; H^{0}\right)$, and every $U_{0, h} \in H^{0}$, there exists a unique solution $U_{h}^{0}$ of (3.10)-(3.11), with $U_{h}^{0} \in \mathcal{C}\left([0, T] ; H^{0}\right), d U_{h} / d t \in L^{1}\left(0, T ; H^{0}\right)$.

\subsection{Convergence as $\varepsilon$ goes to 0 .}

To conclude this section we prove a convergence result as $\varepsilon$ goes to zero of the discrete solution of (3.4)-(3.6) to that of (3.10)-(3.11), $h=\Delta x$ being fixed.

We first endow $H$ with the inner product:

$$
(U, V)_{H}=h \sum_{i=1}^{2 N-2} U_{j} V_{j}+\frac{\varepsilon}{\alpha} U_{2 N-1} V_{2 N-1}+\frac{\varepsilon}{\beta} U_{2 N} V_{2 N}
$$


We have the following discrete integration by parts formula, whose proof is elementary:

Lemma 3.1. Let $\varphi_{h}=\sum_{j=0}^{N} \varphi_{j} w_{j}^{h}$, and $\psi_{h}=\sum_{j=0}^{N} \psi_{j} w_{j}^{h}$.

Then:

$$
\int_{h}^{L-h} \bar{\nabla} \varphi_{h} . \psi_{h} d x=\varphi_{N-1} \psi_{N}-\varphi_{0} \psi_{1}-\int_{h}^{L-h} \varphi_{h} . \nabla \psi_{h} d x
$$

We then observe that the operator $A_{h}$ is semi-definite positive:

Lemma 3.2. For every $U \in H,\left(A_{h} U, U\right)_{H} \geq 0$.

Proof. Let $U=\left(\xi_{1}, \ldots, \xi_{N-1}, \eta_{1}, \ldots, \eta_{N-1}, \xi_{N}, \eta_{0}\right)$ be an element of $D\left(A_{h}\right)=$ $H$. From the definition of the inner product (3.14) on $H$, we find

$$
\begin{aligned}
\left(A_{h} U, U\right)_{H}= & \alpha h \sum_{j=1}^{N-1}(\bar{\nabla} \xi)_{j} \xi_{j}-\beta h \sum_{j=1}^{N-1}(\nabla \eta)_{j} \eta_{j} \\
& -\varepsilon h \sum_{j=1}^{N-1}[\nabla \bar{\nabla}(\xi+\eta)]_{j}(\xi+\eta)_{j} \\
& +\frac{\varepsilon}{h}\left(\xi_{N}-\xi_{N-1}-\eta_{N-1}\right) \xi_{N}-\frac{\varepsilon}{h}\left(\xi_{1}+\eta_{1}-\eta_{0}\right) \eta_{0}
\end{aligned}
$$

Thanks to Lemma 3.1, and using the boundary conditions (3.4) we find

$$
\begin{aligned}
\left(A_{h} U, U\right)_{H}= & \frac{\alpha}{2} \xi_{N-1}^{2}+\frac{\alpha}{2} \sum_{j=1}^{N-1}\left|\xi_{j}-\xi_{j-1}\right|^{2} \\
& +\frac{\beta}{2} \eta_{1}^{2}+\frac{\beta}{2} \sum_{j=1}^{N-1}\left|\eta_{j+1}-\eta_{j}\right|^{2} \\
& +\varepsilon h \sum_{j=1}^{N}\left[\frac{(\xi+\eta)_{j}-(\xi+\eta)_{j-1}}{h}\right]^{2}
\end{aligned}
$$

Finally, we have:

$$
\left(A_{h} U, U\right)_{H} \geq 0
$$

and the lemma is proven.

We now confirm the numerical simulations of [RTT04] with the following result: 
Theorem 3.3. The solutions $U_{h}$ of $(3.4),(3.6)$ converge in $L^{\infty}(0, T ; H)$ to the solution $U_{h}^{0}$ of $(3.10),(3.11)$, when $\varepsilon$ goes to zero.

Proof. We proceed as in Section 2 and set $\tilde{\xi}_{h}=\xi_{h}^{\varepsilon}-\xi_{h}^{0}, \tilde{\eta}_{h}=\eta_{h}^{\varepsilon}-\eta_{h}^{0}$. From equations (3.5),(3.10) and boundary conditions (3.4),(3.6),(3.11) we obtain:

$$
\left\{\begin{aligned}
\frac{d \tilde{\xi}_{j}}{d t}+\alpha(\bar{\nabla} \tilde{\xi})_{j}-\varepsilon[\nabla \bar{\nabla}(\tilde{\xi}+\tilde{\eta})]_{j} & =\varepsilon\left[\nabla \bar{\nabla}\left(\xi^{0}+\eta^{0}\right)\right]_{j} \\
\frac{d \tilde{\eta}_{j}}{d t}-\beta(\nabla \tilde{\eta})_{j}(t)-\varepsilon[\nabla \bar{\nabla}(\tilde{\xi}+\tilde{\eta})]_{j} & =\varepsilon\left[\nabla \bar{\nabla}\left(\xi^{0}+\eta^{0}\right)\right]_{j} \\
\frac{d \tilde{\xi}_{N}}{d t}+\frac{\alpha}{h}\left(\xi_{N}-\xi_{N-1}-\eta_{N-1}\right) & =0 \\
\frac{d \tilde{\eta}_{0}}{d t}-\frac{\beta}{h}\left(\xi_{1}+\eta_{1}-\eta_{0}\right) & =0 .
\end{aligned}\right.
$$

Taking the scalar product of each side of (3.18) with $\bar{U}$ we find after simplification:

$$
\frac{1}{2} \frac{d}{d t} \sum_{j=0}^{N}\left(\tilde{\xi}_{j}^{2}+\tilde{\eta}_{j}^{2}\right) \leq \varepsilon \sum_{j=1}^{N-1}\left|\left[\nabla \bar{\nabla}\left(\xi^{0}+\eta^{0}\right)\right]_{j}\right|\left|\xi_{j}^{0}+\eta_{j}^{0}\right| .
$$

Thanks to the Cauchy-Schwarz inequality, we infer that

$$
\frac{1}{2} \frac{d}{d t} E_{N}(t) \leq E_{N}(t)+K_{2} \varepsilon^{2}
$$

where $E_{N}(t)=\sum_{j=0}^{N}\left(\tilde{\xi}_{j}^{2}(t)+\tilde{\eta}_{j}^{2}(t)\right)$, and $K_{2}$ depends only on $\xi^{0}, \eta^{0}$.

Then we obtain the theorem thanks to the discrete Gronwall lemma.

\section{Conclusion}

In this article we have considered a system of linear PDEs in one space dimension with a small viscosity parameter. This system was derived from the Primitive Equations of the ocean with mild viscosity thanks to a modal decomposition in the vertical direction (see [TT03]). This simple system serves as a model to the study of the important issue of the boundary conditions for the atmosphere and the ocean, in particular for simulations in limited domains. We supplemented the equations with two different sets of boundary conditions, and proved their well-posedness and the convergence of their solutions $\left(u^{\varepsilon}, \psi^{\varepsilon}\right)$ to $\left(u^{0}, \psi^{0}\right)$. The latter is a solution of the limit system obtained by setting 
formally $\varepsilon=0$ in the initial perturbed system.

The first set of boundary conditions is of Dirichlet type and produces some boundary layers and reflections at the boundary. We have also proposed an alternate set of transparent boundary conditions that avoid these reflections. These results are confirmed in the numerical simulations performed in [RTT04].

For the full Primitive Equations, this study suggests that the subcritical and supercritical parts of the solutions need to be considered carefully, in particular regarding the boundary conditions. We leave this to subsequent studies.

\section{Acknowledgements.}

This work was partially supported by the National Science Foundation under the grants NSF-DMS-0074334 and NSF-DMS-0305110, and by the Research Fund of Indiana University. The authors thank David Gottlieb, Stéphane Labbé and Mădălina Petcu for very helpful discussions related to this article. 


\section{REFERENCES}

[Bré73] H. Brézis. Opérateurs maximaux monotones et semi-groupes de contractions dans les espaces de Hilbert. North-Holland Publishing Co., Amsterdam, 1973.

[EM77] B. Engquist and A. Majda. Absorbing boundary conditions for the numerical simulation of waves. Math. Comp., 31(139):629-651, 1977.

[EM79] B. Engquist and A. Majda. Radiation boundary conditions for acoustic and elastic wave calculations. Comm. Pure Appl. Math., 32(3):314-358, 1979 .

[GK79] B. Gustafsson and H.O. Kreiss. Boundary conditions for time-dependent problems with an artificial boundary. J. Comput. Phys., 30(3):333-351, 1979 .

[Got] D. Gottlieb. Personal communication.

[Hal91] L. Halpern. Artificial boundary conditions for incompletely parabolic perturbations of hyperbolic systems. SIAM J. Math. Anal., 22(5):1256$1283,1991$.

[Hen81] D. Henry. Geometric theory of semilinear parabolic equations, volume 840 of Lecture Notes in Mathematics. Springer-Verlag, Berlin, 1981.

[HS89] L. Halpern and M. Schatzman. Artificial boundary conditions for incompressible viscous flows. SIAM J. Math. Anal., 20(2):308-353, 1989.

[Lax86] Peter D. Lax. Hyperbolic systems of conservation laws in several space variables. In Current topics in partial differential equations, pages 327341. Kinokuniya, Tokyo, 1986.

[Lio65] J.L. Lions. Problèmes aux limites dans les équations aux dérivées partielles. Les Presses de l'Université de Montréal, Montreal, Que., 1965. Reedited in [Lio03].

[Lio03] J.L. Lions. Selected work, Vol 1. EDS Sciences, Paris, 2003.

[LR66] J.-L. Lions and P. A. Raviart. Remarques sur la résolution et l'approximation d'équations d'évolution couplées. ICC Bull., 5:1-21, 1966.

[LTW92a] J.L. Lions, R. Temam, and S.H. Wang. New formulations of the primitive equations of atmosphere and applications. Nonlinearity, 5(2):237288, 1992 .

[LTW92b] J.L. Lions, R. Temam, and S.H. Wang. On the equations of the largescale ocean. Nonlinearity, 5(5):1007-1053, 1992.

[Mic85] Daniel Michelson. Initial-boundary value problems for incomplete singular perturbations of hyperbolic systems. In Large-scale computations in fluid mechanics, Part 2 (La Jolla, Calif., 1983), volume 22 of Lectures in Appl. Math., pages 127-132. Amer. Math. Soc., Providence, RI, 1985.

[OS78] J. Oliger and A. Sundström. Theoretical and practical aspects of some initial boundary value problems in fluid dynamics. SIAM J. Appl. Math., 35(3):419-446, 1978.

[Paz83] A. Pazy. Semigroups of operators in Banach spaces. In Equadiff 82 (Würzburg, 1982), volume 1017 of Lecture Notes in Math., pages 508524. Springer, Berlin, 1983.

[Ped87] J. Pedlosky. Geophysical fluid dynamics, 2nd edition. Springer, 1987. 
[RS80] D. Rudy and J. Strikwerda. A nonreflecting outflow boundary condition for subsonic Navier-Stokes calculations. J. Comput. Phys., 36(1):55-70, 1980.

[RS81] D. Rudy and J. Strikwerda. Boundary conditions for subsonic compressible Navier-Stokes calculations. Comput. \& Fluids, 9:327, 1981.

[RTT04] A. Rousseau, R. Temam, and J. Tribbia. Boundary layers in an ocean related system. J. Sci. Comput., 21(3):405-432, 2004.

[Sal98] R. Salmon. Lectures on geophysical fluid dynamics. Oxford University Press, New York, 1998.

[Ser96] Denis Serre. Systèmes de lois de conservation. I, II. Fondations. [Foundations]. Diderot Editeur, Paris, 1996. Hyperbolicité, entropies, ondes de choc. [Hyperbolicity, entropies, shock waves].

[Str77] J. Strikwerda. Initial boundary value problems for incompletely parabolic systems. Comm. Pure Appl. Math., 30(6):797-822, 1977.

[Tem82] R. Temam. Behaviour at time $t=0$ of the solutions of semilinear evolution equations. J. Differential Equations, 43(1):73-92, 1982.

[TH86] L Trefethen and L. Halpern. Well-posedness of one-way wave equations and absorbing boundary conditions. Math. Comp., 47(176):421$435,1986$.

[Tou97] L. Tourrette. Artificial boundary conditions for the linearized compressible Navier-Stokes equations. J. Comput. Phys., 137(1):1-37, 1997.

[TT03] R. Temam and J. Tribbia. Open boundary conditions for the primitive and Boussinesq equations. J. Atmospheric Sci., 60(21):2647-2660, 2003.

[TZ03] R. Temam and M. Ziane. Some mathematical problems in geophysical fluid dynamics. In S. Friedlander and D. Serre, editors, Handbook of mathematical fluid dynamics. North-Holland, 2003.

[WP86] W. Washington and C. Parkinson. An introduction to three-dimensional climate modelling. Oxford Univ. Press, 1986.

[Yos80] K. Yosida. Functional analysis. Springer-Verlag, Berlin, 6th edition, 1980.

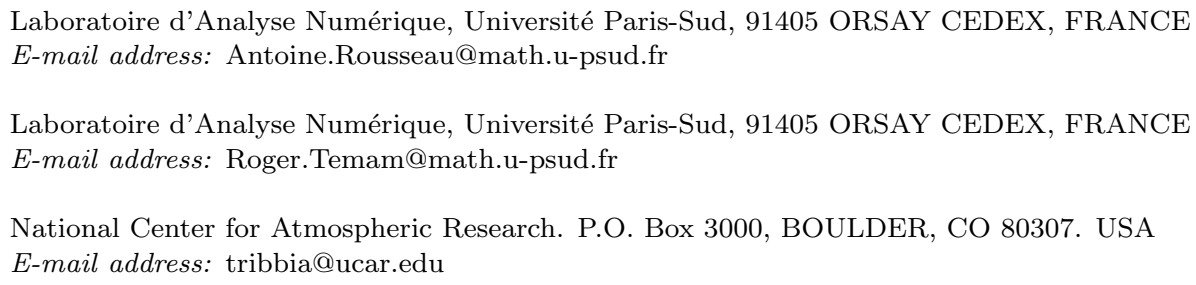

